\title{
Prognostic significance of osteopontin expression in non-small-cell lung cancer: A meta-analysis
}

\author{
XUE-LIN ZOU ${ }^{1 *}, \mathrm{CHUN}_{\text {WANG }}{ }^{* *}, \mathrm{KE} \mathrm{LIU}^{3}, \mathrm{WEN} \mathrm{NIE}^{4}$ and ZHEN-YU DING ${ }^{1}$ \\ ${ }^{1}$ Division of Thoracic Cancer, Cancer Center; ${ }^{2}$ Department of Endocrinology, State Key Laboratory of Biotherapy, \\ West China Hospital, West China Medical School, Sichuan University, Chengdu, Sichuan 610041; ${ }^{3}$ Department \\ of Occupational Health and Radiological Health, Zigong Center for Disease Control and Prevention, Zigong, \\ Sichuan 643000; ${ }^{4}$ School of Clinical Medicine, Sichuan University, Chengdu, Sichuan 610041, P.R. China
}

Received January 8, 2014; Accepted February 9, 2015

DOI: $10.3892 / \mathrm{mco} .2015 .517$

\begin{abstract}
Osteopontin (OPN) plays an important role in the progression and metastasis of cancer. However, the role of OPN as a prognostic factor in non-small-cell lung cancer (NSCLC) remains controversial. The aim of this study was to investigate the association between OPN expression and prognosis in patients with NSCLC using a meta-analysis. Based on PubMed, Ovid Medline, Embase, ISI, ScienceDirect and SpringerLink databases, related articles published prior to January, 2013 were collected. A meta-analysis was conducted to investigate the association of OPN expression with overall survival (OS) and progression-free survival (PFS) in patients with NSCLC. Hazard ratio (HR) with $95 \%$ confidence interval (CI) was used to assess the strength of this association. A total of 6 studies, including 776 patients, were found to be eligible for the meta-analysis. No heterogeneity was observed in OS or PFS, whereas low OPN expression was found to be correlated with better OS (HR=0.57, 95\% CI: 0.46-0.70) and PFS $(\mathrm{HR}=0.62,95 \% \mathrm{CI}: 0.49-0.77)$. This meta-analysis demonstrated an association of OPN with poor prognosis in NSCLC patients. However, prospective studies are required to confirm these findings.
\end{abstract}

\section{Introduction}

Lung cancer ranks first in incidence and mortality worldwide, accounting for 26 and $28 \%$ of cancer-related mortality cases in women and men, respectively (1). The principles of treatment have not changed significantly over several decades

Correspondence to: Dr Zhen-Yu Ding, Division of Thoracic Cancer, Cancer Center, State Key Laboratory of Biotherapy, West China Hospital, West China Medical School, Sichuan University, 37 GuoXue Lane, Chengdu, Sichuan 610041, P.R. China E-mail: dingzy333@163.com

*Contributed equally

Key words: meta-analysis, non-small-cell lung cancer, osteopontin, prognosis and mainly include surgery, radiotherapy or chemotherapy. Recently, targeted therapy was applied to a subset of patients with epithelial growth factor receptor gene mutation or amplification. However, $<20 \%$ of patients with non-small-cell lung cancer (NSCLC) survive for 5 years (2). The identification of prognostic biomarkers is crucial for improving patient outcome. Several prognostic biomarkers have been proposed for NSCLC, such as high excision repair cross-complementation group 1 protein (3), but the results in the literature have been inconsistent.

Osteopontin (OPN) is a multifunctional glycophosphoprotein secreted by different types of cells (4). OPN is an important adhesive bone matrix protein and plays a key role in immune cell recruitment, wound healing and tissue remodeling (5). OPN contains 314 amino acids with extensive post-translational modifications, including phosphorylation and N-linked glycosylation, which result in electrophoretic mobility between 44 and $75 \mathrm{kDa}$. OPN expression is induced during malignant transformation and may contribute to tumorigenesis or, more likely, to the development of a metastatic phenotype (6). OPN expression in NSCLC patients has the potential to provide clinically important prognostic information (7). In the present study, we performed a quantitative meta-analysis of the related published studies to derive a more precise estimation of the significance of OPN.

\section{Materials and methods}

Literature search strategy for identification of related studies. A search was performed, covering all the articles published up to January, 2013, using a combination of the key terms 'osteopontin', 'lung neoplasms' and 'survival analysis' in PubMed and Ovid Medline and a combination of the keywords 'osteopontin' and ('lung neoplasm' or 'lung cancer' or 'lung carcinoma' or 'lung tumor' or 'lung tumour') and ('survival analysis 'or 'survival data') in PubMed, Ovid Medline, ISI, Embase, ScienceDirect and SpringerLink. We evaluated potentially relevant publications by checking the titles and abstracts and procured the most relevant publications for a closer examination. Moreover, the reference lists of the selected articles were also screened for additional articles that were potentially overlooked during the initial search. 
Table I. Newcastle-Ottawa quality assessment scale for cohort studies.

\section{Selection}

1) Representativeness of the exposed cohort

a) Truly representative of the average NSCLC patients in the community ${ }^{\text {a }}$

b) Somewhat representative of the average NSCLC patients in the community ${ }^{a}$

c) Selected group of users e.g., nurses, volunteers

d) No description of the derivation of the cohort

2) Selection of the non exposed cohort

a) Drawn from the same community as the exposed cohort ${ }^{\mathrm{a}}$

b) Drawn from a different source

c) No description of the derivation of the non exposed cohort

3) Ascertainment of exposure
a) Secure record (e.g., surgical records) ${ }^{\mathrm{a}}$
b) Structured interview
c) Written self report
d) No description

4) Demonstration that outcome of interest was not present at start of study
a) $\mathrm{Yes}^{\mathrm{a}}$
b) No

Comparability

1) Comparability of cohorts on the basis of the design or analysis

a) Study controls for recurrence and metastases ${ }^{\mathrm{b}}$

b) Study controls for any additional factor ${ }^{\mathrm{b}}$ (age, gender and grade)

Outcome

1) Assessment of outcome
a) Independent blind assessment ${ }^{\mathrm{a}}$
b) Record linkage
c) Self report
d) No description

2) Was follow-up long enough for outcomes to occur
a) Yes $(\underline{5 \text { years }})^{\mathrm{a}}$
b) No

3) Adequacy of follow-up of cohorts

a) Complete follow-up - all subjects accounted for ${ }^{\mathrm{a}}$

b) Subjects lost to follow-up unlikely to introduce bias - small number lost $\rightarrow \underline{25} \%$ follow-up, or description provided of those lost $\mathrm{t}^{\mathrm{a}}$

c) Follow-up rate $<\underline{25} \%$ and no description of those lost

d) No statement

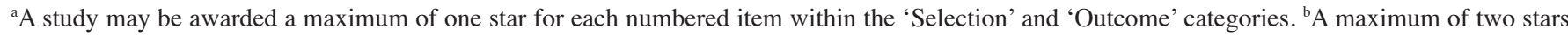
may be awarded for 'Comparability'. Underlined and quoted phrases are provided in the scale to allow for adjustment to particular studies.

The following criteria were used for the study selection: i) The association of OPN levels and the prognosis in patients with NSCLC was considered; ii) the OPN levels were divided on a dichotomy with the median level as the cut-off value in each study; iii) sufficient survival data were provided to calculate hazard ratio (HR) and $95 \%$ confidence interval (CI); iv) the study had long-term follow-up to assess survival; v) OPN expression was measured by immunohistochemistry, enzyme-linked immunosorbent assay, or reverse transcription-quantitative polymerase chain reaction.

Two reviewers (X.-L. Zou and K. Liu) independently screened and retrieved the titles and abstracts of the identified studies and subsequently excluded irrelevant articles. In the case of potentially relevant references, the articles were obtained in full-text format and the following data were extracted from each study: First author's surname, year of publication, disease type, treatment, number of different clinical and pathological parameters and assessment methods of survival expression. Disagreements were resolved through discussion and, when necessary, a third investigator was consulted (W. Nie).

Qualitative assessment. Quality assessment was conducted for each of the available studies by using a generally accepted assessment for cohort studies with moderate modifications (Newcastle-Ottawa quality assessment scale, http://www. ohri.ca/programs/clinical_epidemiology/oxford.asp. Accessed January 1, 2001) (Table I). This scale allows for assessment of patient population and selection, study comparability, follow-up and outcome of interest. The interpretation of the scale is performed by awarding points, or 'stars', for 
Table II. Baseline characteristics of the studies in the meta-analysis.

\begin{tabular}{|c|c|c|c|c|c|c|c|c|c|c|c|c|c|}
\hline $\begin{array}{l}\text { Author } \\
\text { (year) }\end{array}$ & Site & Therapy & Sample & $\begin{array}{c}\text { Median } \\
\text { age } \\
\text { (years) }\end{array}$ & $\begin{array}{l}\text { Tumor } \\
\text { stage }^{\text {a }}\end{array}$ & $\begin{array}{c}\text { Study } \\
\text { quality }^{\mathrm{b}}\end{array}$ & $\begin{array}{l}\text { Sample } \\
\text { size (n) }\end{array}$ & $\begin{array}{l}\text { Detection } \\
\text { method }\end{array}$ & $\begin{array}{l}\text { End- } \\
\text { point }\end{array}$ & HR & $\begin{array}{c}\text { Cut-off } \\
\text { value } \\
(\mathrm{ng} / \mathrm{ml})\end{array}$ & $\begin{array}{c}\text { High } \\
\text { expres- } \\
\text { sion (n) }\end{array}$ & (Refs.) \\
\hline $\begin{array}{l}\text { Takenaka } \\
\text { et al (2013) }\end{array}$ & Jpn & $\begin{array}{l}\text { Surg and } \\
\text { chemo }\end{array}$ & Blood & 69.8 & I-III & $7 / 9$ & 244 & ELISA & OS & NR & 81.3 & 100 & (24) \\
\hline $\begin{array}{l}\text { Isa et al } \\
\text { (2009) }\end{array}$ & Jpn & Chemo & Blood & NR & IIIB-IV & $6 / 9$ & 67 & ELISA & OS/PFS & Reported & 69.0 & 34 & (25) \\
\hline $\begin{array}{l}\text { Mack et al } \\
(2008)\end{array}$ & US & Chemo & Blood & NR & IIIB-IV & $6 / 9$ & 156 & ELISA & OS/PFS & Reported & 592 & 78 & (22) \\
\hline $\begin{array}{l}\text { Le } \text { et al } \\
(2006)\end{array}$ & US & $\begin{array}{l}\text { Surg and } \\
\text { radio }\end{array}$ & Blood & 66 & I-IIIA & $5 / 9$ & 20 & ELISA & PFS & NR & 300 & 7 & (21) \\
\hline $\begin{array}{l}\text { Donati et al } \\
(2005)\end{array}$ & Ita & Surg & Tumor & 65 & I-IIIA & $7 / 9$ & 207 & IHC & OS/PFS & NR & $>20 \%$ & 106 & (19) \\
\hline $\begin{array}{l}\text { Schneider } \\
\text { et al (2004) }\end{array}$ & US & $\begin{array}{l}\text { Surg and } \\
\text { radio }\end{array}$ & Tumor & 62.7 & I-IIIA & $6 / 9$ & 82 & RT-qPCR & OS & NR & 4.06 & 40 & (23) \\
\hline
\end{tabular}

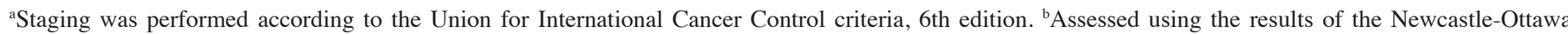
questionnaire (Table I). HR, hazard ratio; Jpn, Japan; surg, surgery; chemo, chemotherapy; ELISA, enzyme-linked immunosorbent assay; OS, overall survival; NR, not reported; PFS, progression-free survival; US, United States; radio, radiotherapy; Ita, Italy; IHC, immunohistochemistry; RT-qPCR, reverse transcription-quantitative polymerase chain reaction.

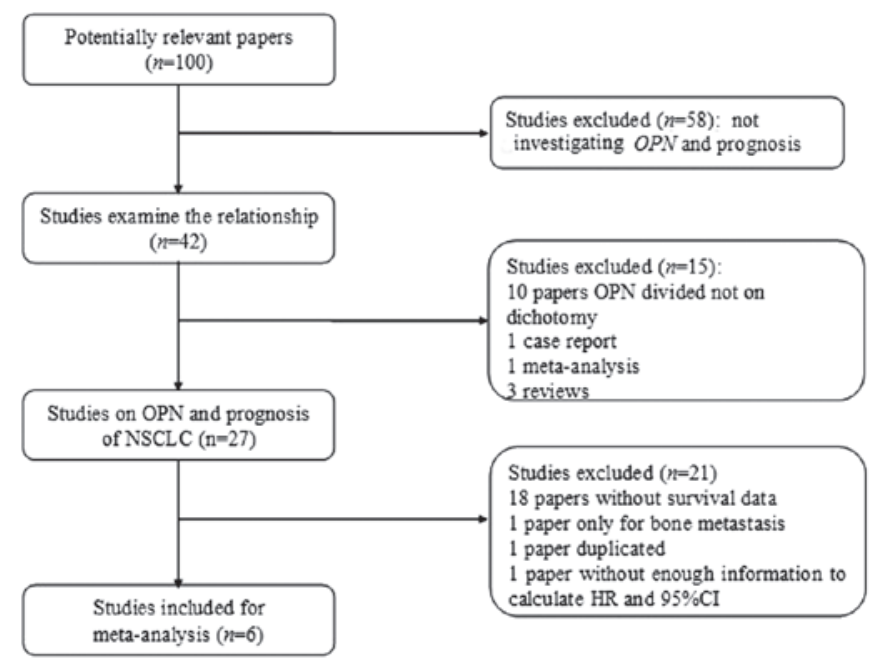

Figure 1. Flow diagram of the study selection process. OPN, osteopontin; NSCLC, non-small-cell lung cancer; HR, hazard ratio; CI, confidence interval.

high-quality elements. Stars are then added up and used to compare study quality in a quantitative manner.

Data extraction. Overall survival (OS) was defined as the interval between the treatment initiation and patient death or last observation. Progression-free survival (PFS) was measured from the date of treatment initiation until the detection of disease recurrence or the last follow-up assessment. From each study, the information summarized in Table II was extracted and tabulated.

Statistical analysis. The included studies were divided into two groups for analysis, those with data regarding OS and those with data on PFS. Data on the predictive ability of OPN (the cut-off value for low vs. high OPN expression was determined by the investigators of each study) for OS and PFS were combined across studies. For the quantitative aggregation of the survival results, HR and 95\% CI were combined to provide the effective value. When these statistical variables were not provided explicitly in an article, they were calculated from available numerical data using the methods reported by Tierney et al (8). The point estimate of the HR was considered statistically significant at $\mathrm{P}<0.05$, provided the $95 \%$ CIs did not include the value 1 . Between-study heterogeneity was determined by performing the $\chi^{2}$-based $Q$ statistics test and was considered significant for $\mathrm{P}<0.10$ (9). The $\mathrm{I}^{2}$ statistic was used as a confirmatory test for heterogeneity, with $\mathrm{I}^{2}<25$, $25-50$ and $>50 \%$ representing low, moderate and high-degree heterogeneity, respectively (10-11). The random-effects model was applied (DerSimonian-Laird method), assuming that studies were sampled from populations with varying effect sizes, calculating the study weights from in-study as well as between-study variances and considering the extent of variation or heterogeneity (12). The fixed-effects model was applied (Mantel-Haenszel method), assuming that the studies were sampled from populations with the same effect size, making an adjustment to the study weights according to the in-study variance (13). An asymmetric Begg's funnel plot suggested possible publication bias and the funnel plot asymmetry was assessed using Egger's linear regression test method (14-15). All the statistical tests were performed using Stata/SE v.12 software, (StataCorp LP, College Station, TX, USA).

\section{Results}

Literature search and meta-analysis database. The search algorithm retrieved 100 references. A total of 58 articles were 
Table III. Publication bias test for prognostic of osteopontin expression in non-small-cell lung cancer.

\begin{tabular}{|c|c|c|c|c|c|}
\hline \multirow[b]{2}{*}{ Survival } & \multicolumn{2}{|c|}{ Begg's test } & \multicolumn{3}{|c|}{ Egger's linear regression test } \\
\hline & $\mathrm{Z}$ & $\mathrm{P}$-value & $\mathrm{t}$ & P-value & Intercept $(95 \% \mathrm{CI})$ \\
\hline Overall & 0.73 & 0.462 & -1.74 & 0.181 & $-2.510(-7.105$ to 2.086$)$ \\
\hline Progression-free & 1.70 & 0.089 & -2.89 & 0.102 & $-2.620(-6.518$ to 1.278$)$ \\
\hline
\end{tabular}
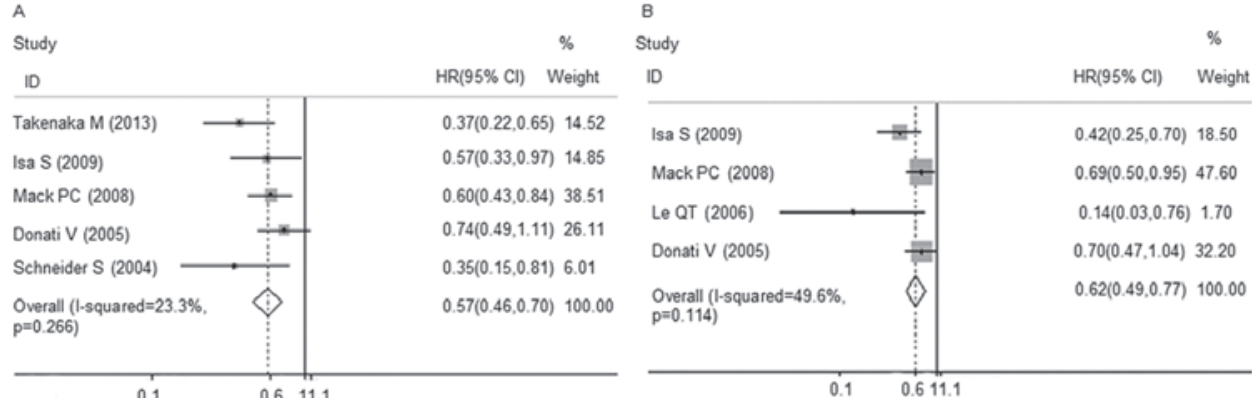

Figure 2. Meta-analysis of the association between osteopontin and (A) overall survival or (B) progression-free survival in patients with non-small-cell lung cancer. HR, hazard ratio; $\mathrm{CI}$, confidence interval.
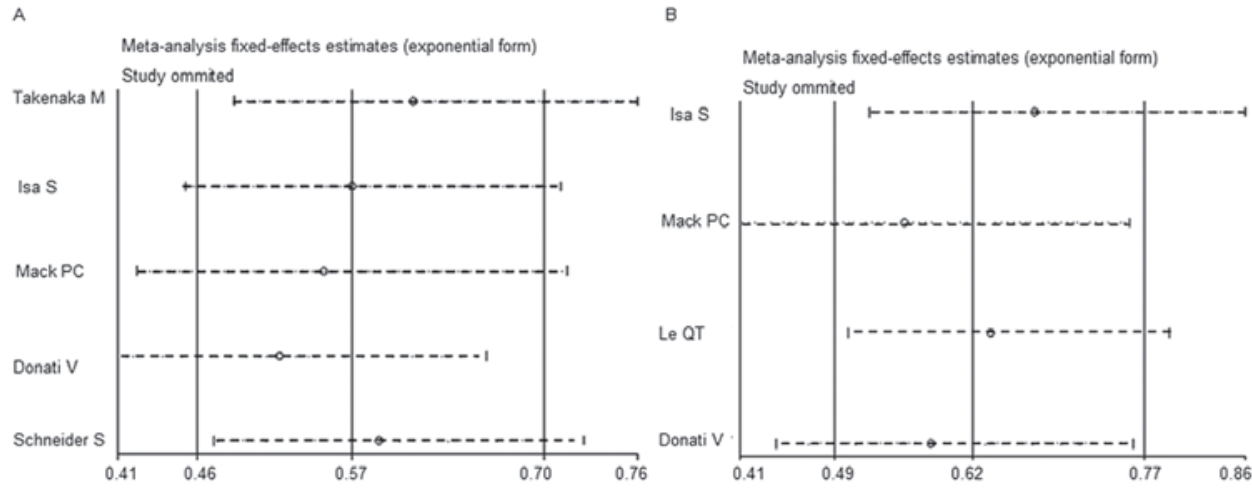

Figure 3. One-way sensitivity analysis of the association between osteopontin and (A) overall survival or (B) progression-free survival in patients with non-small cell lung cancer.

excluded as they were did not investigate the association of OPN and NSCLC; and 10 articles were excluded as the OPN levels were not divided based on dichotomy. One case report and 3 reviews were excluded. A further 18 articles were excluded, as they provided no survival data. Another article was excluded, as it was a meta-analysis comprising only 3 publications on NSCLC not investigating the association between HR and 95\% CI (16).

The remaining 9 studies focused on OPN expression in patients with NSCLC. A further 2 studies were excluded [one focused on the association between OPN and bone metastases (17), whereas the other provided limited data on HR and 95\% CI (18) and the authors were unavailable for more detailed information]. In addition, after performing an in-depth check of the literature, we found that the same cohort was enrolled in two reports $(19,20)$ and only considered the study by Donati et al (19), which had a larger sample size and included more detailed information. Finally, 6 studies were considered eligible for the meta-analysis, comprising a total of 776 patients (Fig. 1). Three of those studies were conducted in America (21-23), two in Japan (24,25) and one in Italy (19). All the studies had a minimum follow-up of 2 years. The study quality determined by the Newcastle-Ottawa quality assessment scale ranged between 5 and 7 (mean, 6.17). Survival was correlated with the OPN expression level, which was assayed with the indicated methods in each study. The characteristics of the eligible studies are summarized in Table II.

Quantitative data synthesis. Among the 6 studies, 5 provided information on OPN expression and OS and 4 on OPN expression and PFS. Low OPN expression was associated with better OS and PFS in the studies, with statistically significant differences. Combined data from the 5 studies demonstrated that low OPN expression was significantly correlated with OS, with a pooled HR estimate of 0.57 (95\% CI: 0.46-0.70, $\mathrm{P}<0.001)$, without heterogeneity in the data $\left(\chi^{2}=5.22, \mathrm{I}^{2}=23.3 \%\right.$, $\mathrm{P}=0.266$; Fig. 2A). Also, combined data from the 4 studies demonstrated that low OPN expression was significantly 
correlated with better PFS, with a pooled HR estimate of 0.62 (95\% CI: 0.49-0.77, $\mathrm{P}<0.001$ ), without heterogeneity in the data $\left(\chi^{2}=5.95, \mathrm{I}^{2}=49.6 \%, \mathrm{P}=0.114\right.$; Fig. 2B).

Sensitivity analysis. In order to compare the differences and evaluate the sensitivity of the meta-analysis, one-way sensitivity analysis was conducted to evaluate the stability of the meta-analysis (Fig. 3). The $\mathrm{I}^{2}$ value was not significantly affected when any single study was omitted, with the statistical significance of the overall effect size remaining unchanged. Therefore, the results of the sensitivity analysis suggested that the data in this meta-analysis were stable and reliable.

Publication bias. Funnel plots were created for the assessment of possible publication bias. Egger's linear regression test was used for the quantitative evaluation of the symmetry of the meta-analysis funnel plot (Table III). The 95\% CI of intercept included zero in Egger's publication bias plot, suggesting that the results of this meta-analysis are relatively stable and not significantly affected by publication bias.

\section{Discussion}

The identification of biomarkers is crucial for guiding clinical decision-making. Biomarkers provide valuable information on potential therapeutic benefits. For example, the 21-gene signature helps to prescribe adjuvant chemotherapy for breast cancer patients who would be difficult to assess based on clinical characteristics alone (26). The presence of K-ras mutations strongly argues against administering cetuximab to colon cancer patients (27). Prognostic biomarkers are also being extensively investigated for NSCLC. A 14-gene signature was proposed as a prognostic factor for early-phase NSCLC (28). However, no prognostic biomarkers are currently available for NSCLC in daily practice and biomarker research is urgently required.

OPN has been suggested as a candidate prognostic marker for multiple malignancies. Chambers et al (7) suggested its prognostic value and other groups also supported this idea $(22,25)$. Although the association between OPN and prognosis has been investigated over several years, no definitive conclusion has been drawn. Considering that a meta-analysis is a valuable tool in biomarker validation, we conducted the present meta-analysis to investigate the association between OPN expression and the prognosis of NSCLC.

In the present study, we identified 6 articles, including a total of 776 NSCLC patients, analyzing the association between OPN and prognosis. The study quality was determined using the Newcastle-Ottawa method. The pooled results demonstrated that low OPN expression was significantly associated with better OS (0.57, 95\% CI: 0.46-0.70) and PFS (0.62, 95\% CI: $0.49-0.77)$. The sensitivity analysis suggested that the data in this meta-analysis were stable. The quantitative evaluation of the symmetry of the meta-analysis funnel plot suggested the publication bias did not significantly affect the results of the meta-analysis.

An association between OPN and prognosis of NSCLC was previously suggested (16). However, there remained controversies regarding its prognostic value. For example, one study argued the OPN expression was not associated with OS or PFS (19). As the P-values in that study exhibited a strong tendency toward statistical significance $(\mathrm{P}=0.14$ and $\mathrm{P}=0.074$, respectively), it may be reasoned that the conclusion was due to an insufficient sample size. Compared to previous reports, our meta-analysis enrolled more patients $(n=776)$ compared to any individual study. Meta-analyses such as the present may help solve the problem of inconsistency and consolidate the results. In addition, our study quantitated the prognostic value of OPN by providing the HR and $95 \%$ CI. Therefore, our study strongly supports that OPN expression is negatively correlated with prognosis in NSCLC.

The mechanisms underlying the aggressive phenotype associated with OPN expression in tumors have been extensively investigated. OPN on the surface of a wide spectrum of malignant cells was reported to recognize and ligate integrins or CD44 and activate intracellular signaling, such as the phosphoinositide 3-kinase or mitogen-activated protein kinase pathways (29). As a consequence, various transcription factors, including nuclear factor- $\kappa \mathrm{B}$ or activator protein-1 are activated and the expression level of factors inhibiting apoptosis and promoting matrix degradation is increased. Consistent with clinical data, an in vitro study demonstrated that the overexpression of OPN promoted the malignant phenotype, whereas its knockdown yielded a population with a reduced malignant potential (30). These studies may help interpret the observations in our study.

It should be noted that our study had certain limitations. Although the method used to estimate HR and 95\% CI is a method universally adopted (31), it was a univariate analysis and publication bias was inevitable, as the unpublished data was not included. Finally, the detection methods were different across studies. However, the data in our meta-analysis strongly suggest that OPN expression is negatively associated with prognosis in patients with NSCLC, although its prognostic value merits further validation.

\section{Acknowledgements}

This study was supported by grants from the National Major Project of China (no. 2011ZX09302-001-01) and the National Natural Science Foundation of China (nos. 81272684 and 81200640).

\section{References}

1. Siegel R, Naishadham D and Jemal A: Cancer statistics, 2013: CA Cancer J Clin 63: 11-30, 2013.

2. Spiro SG and Silvestri GA: One hundred years of lung cancer. Am J Respir Crit Care Med 172: 523-529, 2005.

3. Zheng Z, Chen T, Li X, Haura E, Sharma A and Bepler G: DNA synthesis and repair genes RRM1 and ERCC1 in lung cancer. N Engl J Med 356: 800-808, 2007.

4. Denhardt DT and Guo X: Osteopontin: a protein with diverse functions. FASEB J 7: 1475-1482, 1993.

5. Shijubo N, Kojima H, Nagata M, Ohchi T, Suzuki A, Abe S and Sato N: Tumor angiogenesis of non-small cell lung cancer. Microsc Res Tech 60: 186-198, 2003.

6. O'Regan A: The role of osteopontin in lung disease. Cytokine Growth Factor Rev 14: 479-488, 2003

7. Chambers AF, Wilson SM, Kerkvliet N, O'Malley FP, Harris JF and Casson AG: Osteopontin expression in lung cancer. Lung Cancer 15: 311-323, 1996.

8. Tierney JF, Stewart LA, Ghersi D, Burdett S and Sydes MR: Practical methods for incorporating summary time-to-event data into meta-analysis. Trials 8: 16, 2007. 
9. Whitehead A and Whitehead J: A general parametric approach to the meta-analysis of randomized clinical trials. Stat Med 10 $1665-1677,1991$.

10. Ioannidis JP, Patsopoulos NA and Evangelou E: Uncertainty in heterogeneity estimates in meta-analyses. BMJ 335: 914-916, 2007.

11. Higgins JP, Thompson SG, Deeks JJ and Altman DG: Measuring inconsistency in meta-analyses. BMJ 327: 557-560, 2003.

12. DerSimonian R and Laird N: Meta-analysis in clinical trials. Control Clin Trials 7: 177-188, 1986.

13. Mantel N and Haenszel W: Statistical aspects of the analysis of data from retrospective studies of disease. J Natl Cancer Inst 22: 719-748, 1959.

14. Egger M, Davey Smith G, Schneider M and Minder C: Bias in meta-analysis detected by a simple, graphical test. BMJ 315: 629-634, 1997.

15. Macaskill P, Walter SD and Irwig L: A comparison of methods to detect publication bias in meta-analysis. Stat Med 20: 641-654, 2001.

16. Weber GF, Lett GS and Haubein NC: Osteopontin is a marker for cancer aggressiveness and patient survival. Br J Cancer 103: $861-869,2010$

17. Zhang L, Hou X, Lu S, et al: Predictive significance of bone sialoprotein and osteopontin for bone metastases in resected Chinese non-small-cell lung cancer patients: a large cohort retrospective study. Lung Cancer 67: 114-119, 2010.

18. Shijubo N, Uede T, Kon S, Maeda M, Segawa T, Imada A Hirasawa $\mathrm{M}$ and Abe S: Vascular endothelial growth factor and osteopontin in stage I lung adenocarcinoma. Am J Respir Crit Care Med 160: 1269-1273, 1999.

19. Donati V, Boldrini L, Dell'Omodarme M, Prati MC, Faviana P, Camacci T, Lucchi M, Mussi A, Santoro M, Basolo F and Fontanini G: Osteopontin expression and prognostic significance in non-small cell lung cancer. Clin Cancer Res 11: 6459-6465, 2005.

20. Boldrini L, Donati V, Dell'Omodarme M, Prati MC, Faviana P, Camacci T, Lucchi M, Mussi A, Santoro M, Basolo F and Fontanini G: Prognostic significance of osteopontin expression in early-stage non-small-cell lung cancer. Br J Cancer 93 453-457, 2005

21. Le QT, Chen E, Salim A, et al: An evaluation of tumor oxygenation and gene expression in patients with early stage non-small cell lung cancers. Clin Cancer Res 12: 1507-1514, 2006.
22. Mack PC, Redman MW, Chansky K, Williamson SK, Farneth NC, Lara PN Jr, Franklin WA, Le QT, Crowley JJ, Gandara DR and SWOG: Lower osteopontin plasma levels are associated with superior outcomes in advanced non-small-cell lung cancer patients receiving platinum-based chemotherapy: SWOG Study S0003. J Clin Oncol 26: 4771-4776, 2008.

23. Schneider S, Yochim J, Brabender J, Uchida K, Danenberg KD, Metzger R, Schneider PM, Salonga D, Hölscher AH and Danenberg PV: Osteopontin but not osteonectin messenger RNA expression is a prognostic marker in curatively resected non-small cell lung cancer. Clin Cancer Res 10: 1588-1596, 2004.

24. Takenaka M, Hanagiri T, Shinohara S, et al: Serum level of osteopontin as a prognostic factor in patients who underwent surgical resection for non-small-cell lung cancer. Clin Lung Cancer 14: 288-294, 2013.

25. Isa S, Kawaguchi T, Teramukai S, et al: Serum osteopontin levels are highly prognostic for survival in advanced non-small cell lung cancer: results from JMTO LC 0004. J Thorac Oncol 4: 1104-1110, 2009

26. Albain KS, Barlow WE, Shak S, et al: Prognostic and predictive value of the 21-gene recurrence score assay in postmenopausal women with node-positive, estrogen-receptor-positive breast cancer on chemotherapy: a retrospective analysis of a randomised trial. Lancet Oncol 11: 55-65, 2010.

27. Lièvre A, Bachet JB, Boige V, et al: KRAS mutations as an independent prognostic factor in patients with advanced colorectal cancer treated with cetuximab. J Clin Oncol 26: 374-379, 2008.

28. Kratz JR, He J, Van Den Eeden SK, et al: A practical molecular assay to predict survival in resected non-squamous, non-small-cell lung cancer: development and international validation studies. Lancet 379: 823-832, 2012.

29. Rangaswami H, Bulbule A and Kundu GC: Osteopontin: role in cell signaling and cancer progression. Trends Cell Biol 16: 79-87, 2006.

30. Goparaju CM, Pass HI, Blasberg JD, Hirsch N and Donington JS: Functional heterogeneity of osteopontin isoforms in non-small cell lung cancer. J Thorac Oncol 5: 1516-1523, 2010.

31. Rahbari NN, Aigner M, Thorlund K, et al: Meta-analysis shows that detection of circulating tumor cells indicates poor prognosis in patients with colorectal cancer. Gastroenterology 138: 1714-1726, 2010 . 